\title{
Mapping Properties of Generalized Robertson Functions under Certain Integral Operators
}

\author{
Muhammad Arif*, Wasim Ul-Haq, Muhammad Ismail \\ Department of Mathematics, Abdul Wali Khan University, Mardan, Pakistan \\ Email: \{marifmaths, ismail1350\}@yahoo.com,wasim474@hotmail.com
}

Received July 24, 2011; revised November 24, 2011; accepted December 2, 2011

\section{ABSTRACT}

In the present article, certain classes of generalized $p$-valent Robertson functions are considered. Mapping properties of these classes are investigated under certain $p$-valent integral operators introduced by Frasin recently.

Keywords: $p$-Valent Analytic Functions; Bounded Boundary Rotations; Bounded Radius Rotations; Integral Operators

\section{Introduction}

Let $A(p)$ be the class of functions $f(z)$ of the form

$$
f(z)=z^{p}+\sum_{j=p+1}^{\infty} a_{j} z^{j}(p \in \mathbb{N}=\{1,2, \cdots\}),
$$

which are analytic in the open unit disc $E=\{z:|z|<1\}$. We write $A(1)=A$. A function $f(z) \in A$ is said to be spiral-like if there exists a real number $\lambda \quad\left(|\lambda|<\frac{\pi}{2}\right)$ such that

$$
\operatorname{Re} \mathrm{e}^{i \lambda} \frac{z f^{\prime}(z)}{f(z)}>0(z \in E) .
$$

The class of all spiral-like functions was introduced by L. Spacek [1] in 1933 and we denote it by $S_{\lambda}^{*}$. Later in
1969, Robertson [2] considered the class $C_{\lambda}$ of analytic functions in $E$ for which $z f^{\prime}(z) \in S_{\lambda}^{*}$.

Let $P_{k}^{\lambda}(p, \rho)$ be the class of functions $p(z)$ analytic in $E$ with $p(0)=1$ and

$$
\int_{0}^{2 \pi}\left|\frac{\operatorname{Re} \mathrm{e}^{i \lambda} p(z)-\rho \cos \lambda}{p-\rho}\right| \mathrm{d} \theta \leq k \pi \cos \lambda,\left(z=r \mathrm{e}^{i \theta}\right),
$$

where $k \geq 2,0 \leq \rho<1$ and $\lambda$ is real with $|\lambda|<\frac{\pi}{2}$.

For $\lambda=0, p=1$, this class was introduced in [3] and for $\rho=0$, see [4]. For $k=2, \lambda=0$ and $\rho=0$, the class $P_{k}^{\lambda}(p, \rho)$ reduces to the class $P$ of functions $p(z)$ analytic in $E$ with $p(0)=1$ and whose real part is positive.

We define the following classes

$$
\begin{gathered}
R_{k}^{\lambda}(p, \rho)=\left\{f(z): f(z) \in A(p) \text { and } \frac{z f^{\prime}(z)}{f(z)} \in P_{k}^{\lambda}(p, \rho), 0 \leq \rho<1\right\}, \\
V_{k}^{\lambda}(p, \rho)=\left\{f(z): f(z) \in A(p) \text { and } \frac{\left(z f^{\prime}(z)\right)^{\prime}}{f^{\prime}(z)} \in P_{k}^{\lambda}(p, \rho), 0 \leq \rho<1\right\} .
\end{gathered}
$$

For $\lambda=0, \rho=0$ and $p=1$, we obtain the well known classes $R_{k}$ and $V_{k}$ of analytic functions with bounded radius and bounded boundary rotations studied by Tammi [5] and Paatero [6] respectively. For details see [7-12]. Also it can easily be seen that $R_{2}^{\lambda}(0)=S_{\lambda}^{*}$ and $V_{2}^{\lambda}(0)=C_{\lambda}$.

Let us consider the integral operators

\footnotetext{
${ }^{*}$ Corresponding author.
}

$$
F_{p}(z)=\int_{0}^{z} p t^{p-1}\left(\frac{f_{1}(t)}{t^{p}}\right)^{\alpha_{1}} \cdots\left(\frac{f_{n}(t)}{t^{p}}\right)^{\alpha_{n}} \mathrm{~d} t
$$

and

$$
G_{p}(z)=\int_{0}^{z} p t^{p-1}\left[\frac{f_{1}^{\prime}(t)}{p t^{p-1}}\right]^{\alpha_{1}} \ldots\left[\frac{f_{n}^{\prime}(t)}{p t^{p-1}}\right]^{\alpha_{n}} \mathrm{~d} t,
$$

where $f_{i}(z) \in A(p)$ and $\alpha_{i}>0$ for all $i \in\{1,2, \cdots, n\}$. 
These operators, given by (1.1) and (1.2), are defined by Frasin [13]. If we take $p=1$, we obtain the integral operators $F_{1}(z)=F_{n}(z)$ and $G_{1}(z)=F_{\alpha_{1} \cdots \alpha_{n}}(z)$ introduced and studied by Breaz and Breaz [14] and Breaz et al. [15], for details see also [16-20]. Also for $p=n=1$, $\alpha_{1}=\alpha \in[0,1]$ in (1.1), we obtain the integral operator studied in [21] given as

$$
\int_{0}^{z}\left(\frac{f(t)}{t}\right)^{\alpha} \mathrm{d} t
$$

and for $p=n=1, \quad \alpha_{1}=\delta \in \mathbb{C},|\delta| \leq \frac{1}{4}$ in (1.2), we obtain the integral operator

$$
\int_{0}^{z}\left(f^{\prime}(t)\right)^{\delta} \mathrm{d} t
$$

discussed in [22,23].

In this paper, we investigate some propeties of the above integral operators $F_{p}(z)$ and $G_{p}(z)$ for the classes $V_{k}^{\lambda}(p, \rho)$ and $R_{k}^{\lambda}(p, \rho)$ respectively.

\section{Main Result}

Theorem 2.1. Let $f_{i}(z) \in R_{k}^{\lambda}(p, \rho)$ for $1 \leq i \leq n$ with $0 \leq \rho<1$. Also let $\lambda$ is real with $|\lambda|<\frac{\pi}{2}, \alpha_{i}>0$, $1 \leq i \leq n$. If

$$
0 \leq(\rho-p) \sum_{i=1}^{n} \alpha_{i}+p<1,
$$

then $F_{p}(z) \in V_{k}^{\lambda}\left(p, \lambda_{1}\right)$ with

$$
\lambda_{1}=(\rho-p) \sum_{i=1}^{n} \alpha_{i}+p
$$

Proof. From (1.1), we have

$$
\frac{z F_{p}^{\prime \prime}(z)}{F_{p}^{\prime}(z)}=(p-1)+\sum_{i=1}^{n} \alpha_{i}\left(\frac{z f_{i}^{\prime}(z)}{f_{i}(z)}-p\right),
$$

or, equivalently

$$
\mathrm{e}^{i \lambda}\left(1+\frac{z F_{p}^{\prime \prime}(z)}{F_{p}^{\prime}(z)}\right)=\mathrm{e}^{i \lambda} p\left(1-\sum_{i=1}^{n} \alpha_{i}\right)+\mathrm{e}^{i \lambda} \sum_{i=1}^{n} \alpha_{i} \frac{z f_{i}^{\prime}(z)}{f_{i}(z)} .
$$

Subtracting and adding $\rho \cos \lambda \sum_{i=1}^{n} \alpha_{i}$ on the right hand side of (2.3), we have

$$
\begin{aligned}
\mathrm{e}^{i \lambda}\left(1+\frac{z F_{p}^{\prime \prime}(z)}{F_{p}^{\prime}(z)}\right)= & p \mathrm{e}^{i \lambda}+\left(\rho \cos \lambda-p \mathrm{e}^{i \lambda}\right) \sum_{i=1}^{n} \alpha_{i} \\
& +\sum_{i=1}^{n} \alpha_{i}\left[\mathrm{e}^{i \lambda} \frac{z f_{i}^{\prime}(z)}{f_{i}(z)}-\rho \cos \lambda\right],
\end{aligned}
$$

Taking real part of (2.4) and then simple computation gives

$$
\begin{aligned}
& \int_{0}^{2 \pi} \mid \operatorname{Re}\left[\mathrm{e}^{i \lambda}\left(1+\frac{z F_{p}^{\prime \prime}(z)}{F_{p}^{\prime}(z)}-\lambda_{1} \cos \lambda\right] \mid \mathrm{d} \theta\right. \\
& \leq \sum_{i=1}^{n} \alpha_{i} \int_{0}^{2 \pi}\left|\operatorname{Re}\left[\mathrm{e}^{i \lambda} \frac{z f_{i}^{\prime}(z)}{f_{i}(z)}-\rho \cos \lambda\right]\right| \mathrm{d} \theta,
\end{aligned}
$$

where $\lambda_{1}$ is given by (2.1). Since $f_{i}(z) \in R_{k}^{\lambda}(p, \rho)$ for $1 \leq i \leq n$, we have

$$
\int_{0}^{2 \pi}\left|\operatorname{Re}\left[\mathrm{e}^{i \lambda} \frac{z f_{i}^{\prime}(z)}{f_{i}(z)}-\rho \cos \lambda\right]\right| \mathrm{d} \theta \leq(p-\rho) \cos \lambda k \pi .
$$

Using (2.6) and (2.1) in (2.5), we obtain

$$
\int_{0}^{2 \pi} \mid \operatorname{Re}\left[\mathrm{e}^{i \lambda}\left(1+\frac{z F_{p}^{\prime \prime}(z)}{F_{p}^{\prime}(z)}\right)-\lambda_{1} \cos \lambda\right] \mathrm{d} \theta \leq\left(p-\lambda_{1}\right) \cos \lambda k \pi .
$$

Hence $F_{n}(z) \in V_{k}^{\lambda}\left(p, \lambda_{1}\right)$ with $\lambda_{1}$ is given by (2.1). By setting $p=1$ and $\lambda=0$ in Theorem 2.1, we obtain the following result proved in [9].

Corollory 2.2. Let $f_{i}(z) \in R_{k}(\rho)$ for $1 \leq i \leq n$ with $0 \leq \rho<1$. Also let $\alpha_{i}>0,1 \leq i \leq n$. If

$$
0 \leq(\rho-1) \sum_{i=1}^{n} \alpha_{i}+1<1,
$$

then $F_{n}(z) \in V_{k}\left(\lambda_{1}\right)$ and $\lambda_{1}$ is given by (2.1).

Now if we take $k=2$ and $\lambda=0$ in Theorem 2.1, we obtain the following result.

Corollory 2.3. Let $f_{i}(z) \in S_{p}^{*}(\rho)$ for $1 \leq i \leq n$ with $0 \leq \rho<1$. Also let $\alpha_{i}>0,1 \leq i \leq n$. If

$$
0 \leq(\rho-p) \sum_{i=1}^{n} \alpha_{i}+p<1,
$$

then $F_{p}(z) \in C_{p}\left(\lambda_{1}\right)$ and $\lambda_{1}$ is given by (2.1).

Letting $p=n=1, \lambda=0, \alpha_{1}=\alpha$ and $f_{1}(z)=f(z)$ in Theorem 2.1, we have.

Corollory 2.4. Let $f(z) \in R_{k}(\rho)$ with $0 \leq \rho<1$. Also let $\alpha>0$. If

$$
0 \leq(\rho-1) \alpha+1<1,
$$

then

$$
\int_{0}^{z}\left(\frac{f(t)}{t}\right)^{\alpha} \mathrm{d} t \in V_{k}\left(\lambda_{1}\right)
$$

with $\lambda_{1}=(\rho-1) \alpha+1$.

Theorem 2.5. Let $f_{i}(z) \in V_{k}^{\lambda}(p, \rho)$ for $1 \leq i \leq n$ with $0 \leq \rho<1$. Also let $\lambda$ is real is real with $|\lambda|<\frac{\pi}{2}$, $\alpha_{i}>0,1 \leq i \leq n$. If 


$$
0 \leq(\rho-p) \sum_{i=1}^{n} \alpha_{i}+p<1,
$$

then $G_{p}(z) \in V_{k}^{\lambda}\left(p, \lambda_{1}\right)$ and $\lambda_{1}$ is given by (2.1).

Proof. From (1.2), we have

$$
1+\frac{z G_{p}^{\prime \prime}(z)}{G_{p}^{\prime}(z)}=p+\sum_{i=1}^{n} \alpha_{i}\left(\frac{z f_{i}^{\prime \prime}(z)}{f_{i}^{\prime}(z)}+1\right)-p \sum_{i=1}^{n} \alpha_{i},
$$

or, equivalently

$$
\begin{aligned}
& \mathrm{e}^{i \lambda}\left(1+\frac{z G_{p}^{\prime \prime}(z)}{G_{p}^{\prime}(z)}\right) \\
& =p \mathrm{e}^{i \lambda}\left(1-\sum_{i=1}^{n} \alpha_{i}\right)+\sum_{i=1}^{n} \alpha_{i} \mathrm{e}^{i \lambda}\left(1+\frac{z f_{i}^{\prime \prime}(z)}{f_{i}^{\prime}(z)}\right) .
\end{aligned}
$$

This relation is equivalent to

$$
\begin{aligned}
& \mathrm{e}^{i \lambda}\left(1+\frac{z G_{p}^{\prime \prime}(z)}{G_{p}^{\prime}(z)}\right)=p \mathrm{e}^{i \lambda}+\left(\rho \cos \lambda-p \mathrm{e}^{i \lambda}\right) \sum_{i=1}^{n} \alpha_{i} \\
& +\sum_{i=1}^{n} \alpha_{i}\left[\mathrm{e}^{i \lambda}\left(1+\frac{z f_{i}^{\prime \prime}(z)}{f_{i}^{\prime}(z)}\right)-\rho \cos \lambda\right] .
\end{aligned}
$$

Taking real part of (2.7) and then simple computation gives us

$$
\begin{aligned}
& \int_{0}^{2 \pi} \mid \operatorname{Re}\left[\mathrm{e}^{i \lambda}\left(1+\frac{z G_{p}^{\prime \prime}(z)}{G_{p}^{\prime}(z)}\right)-\lambda_{1} \cos \lambda\right] \mathrm{d} \theta \\
& \leq \sum_{i=1}^{n} \alpha_{i} \int_{0}^{2 \pi} \mid \operatorname{Re}\left[\mathrm{e}^{i \lambda}\left(1+\frac{z f_{i}^{\prime \prime}(z)}{f_{i}^{\prime}(z)}\right)-\rho \cos \lambda\right] \mathrm{d} \theta,
\end{aligned}
$$

where $\lambda_{1}$ is given by (2.1). Since $f_{i}(z) \in V_{k}^{\lambda}(p, \rho)$ for $1 \leq i \leq n$, we have

$$
\int_{0}^{2 \pi}\left|\operatorname{Re}\left[\mathrm{e}^{i \lambda}\left(1+\frac{z f_{i}^{\prime \prime}(z)}{f_{i}^{\prime}(z)}\right)-\rho \cos \lambda\right]\right| \mathrm{d} \theta \leq(p-\rho) \cos \lambda k \pi .
$$

Using (2.9) in (2.8), we obtain

$$
\int_{0}^{2 \pi}\left|\operatorname{Re}\left[\mathrm{e}^{i \lambda}\left(1+\frac{z G_{p}^{\prime \prime}(z)}{G_{p}^{\prime}(z)}\right)-\lambda_{1} \cos \lambda\right]\right| \mathrm{d} \theta \leq\left(p-\lambda_{1}\right) \cos \lambda k \pi .
$$

Hence $G_{p}(z) \in V_{k}^{\lambda}\left(p, \lambda_{1}\right)$ with $\lambda_{1}$ is given by (2.1).

By setting $k=2$ and $\lambda=0$ in Theorem 2.5, we obtain the following result.

Corollory 2.6. Let $f_{i}(z) \in C_{p}(\rho)$ for $1 \leq i \leq n$ with $0 \leq \rho<1$. Also let $\alpha_{i}>0,1 \leq i \leq n$. If

$$
0 \leq(\rho-p) \sum_{i=1}^{n} \alpha_{i}+p<1,
$$

then $G_{p}(z) \in C_{p}\left(\lambda_{1}\right)$ with $\lambda_{1}$ is given by (2.1).

Letting $p=n=1, \lambda=0, \alpha_{1}=\delta$ and $f_{1}(z)=f(z)$ in Theorem 2.5, we have.

Corollory 2.7. Let $f(z) \in V_{k}(\rho)$ with $0 \leq \rho<1$. Also let $\delta>0$. If $0 \leq(\rho-1) \delta+1<1$, then

$$
\int_{0}^{z}\left(f^{\prime}(t)\right)^{\delta} \mathrm{d} t \in V_{k}\left(\lambda_{1}\right)
$$

with $\lambda_{1}=(\rho-1) \delta+1$.

\section{REFERENCES}

[1] L. Spacek, "Prispěvek k Teorii Funkei Prostych," Časopis pro pěstováni matematiky a fysiky, Vol. 62, No. 2, 1933, pp. 12-19.

[2] M. S. Robertson, "Univalent Functions $f(z)$ for wich $z f^{\prime}(z)$ Is Spiral-Like," Michigan Mathematical Journal, Vol. 16, No. 2, 1969, pp. 97-101.

[3] K. S. Padmanabhan and R. Parvatham, "Properties of a Class of Functions with Bounded Boundary Rotation," Annales Polonici Mathematici, Vol. 31, No. 1, 1975, pp. 311-323.

[4] B. Pinchuk, "Functions with Bounded Boundary Rotation," Israel Journal of Mathematics, Vol. 10, No. 1, 1971, pp. 7-16. doi:10.1007/BF02771515

[5] O. Tammi, "On the Maximization of the Coefficients of Schlicht and Related Functions," Annales Academiae Scientiarum Fennicae. Series A I. Mathematica, Vol. 114, No. 1, 1952, p. 51

[6] V. Paatero, "Uber Gebiete von Beschrankter Randdrehung," Annales Academiae Scientiarum Fennnicae, Vol. 37-39, No. 9, 1933.

[7] M. Arif, M. Ayaz and S. I. Ali Shah, "Radii Problems for Certain Classes of Analytic Functions with Fixed Second Coefficients," World Applied Sciences Journal, Vol. 13, No. 10, 2011, pp. 2240-2243.

[8] K. I. Noor, "On Some Subclasses of Fuctions with Bounded Boundary and Bounded Radius Rotation," Pan American Mathematical Journal, Vol. 6, No. 1, 1996, pp. 75-81.

[9] K. I. Noor, M. Arif and W. Haq, "Some Properties of Certain Integral Opertors," Acta Universitatis Apulensis, Vol. 21, 2010, pp. 89-95.

[10] K. I. Noor, M. Arif and A. Muhammad, "Mapping Properties of Some Classes of Analytic Functions under an Integral Operator," Journal of Mathematical Inequalities, Vol. 4, No. 4, 2010, pp. 593-600.

[11] K. I. Noor, W. Haq, M. Arif and S. Mustafa, "On Bounded Boundary and Bounded Radius Rotations," Journal of Inequalities and Applications, 2009, Article ID: 813687.

[12] K. I. Noor, S. N. Malik, M. Arif and M. Raza, "On Bounded Boundary and Bounded Radius Rotation Related with Janowski Function," World Applied Sciences Journal, Vol. 12, No. 6, 2011, pp. 895-902.

[13] B. A. Frasin, "New General Integral Operators of $p$-Valent Functions," Journal of Inequatilies Pure and Applied Mathematics, Vol. 10, No. 4, 2009

[14] D. Breaz and N. Breaz, "Two Integral Operators," Studia Universitatis Babes-Bolyai, Mathematica, Clunj-Napoca, 
Vol. 47, No. 3, 2002, pp. 13-21.

[15] D. Breaz, S. Owa and N. Breaz, "A New Integral Univalent Operator," Acta Universitatis Apulensis, Vol. 16, 2008, pp. 11-16.

[16] N. Breaz, V. Pescar and D. Breaz, "Univalence Criteria for a New Integral Operator," Mathematical and Computer Modelling, Vol. 52, No. 1-2, 2010, pp. 241-246. doi:10.1016/j.mcm.2010.02.013

[17] B. A. Frasin, "Convexity of Integral Operators of $p$-Valent Functions," Mathematical and Computer Modelling, Vol. 51, No. 5-6, 2010, pp. 601-605.

[18] B. A. Frasin, "Some Sufficient Conditions for Certain Integral Operators," Journal of Mathematics and Inequalities, Vol. 2. No. 4, 2008, pp. 527-535.

[19] G. Saltik, E. Deniz and E. Kadioglu, "Two New General p-Valent Integral Operators," Mathematical and Compu- ter Modelling, Vol. 52, No. 9-10, 2010, pp. 1605-1609. doi:10.1016/j.mcm.2010.06.025

[20] R. M. Ali and V. Ravichandran, "Integral Operators on Ma-Minda Type Starlike and Convex Functions," Mathematical and Computer Modelling, Vo. 53, No. 5-6, 2011, pp. 581-586. doi:10.1016/j.mcm.2010.09.007

[21] S. S. Miller, P. T. Mocanu and M. O. Reade, "Starlike Integral Operators," Pacific Journal of Mathematics, Vol. 79, No. 1, 1978, pp.157-168.

[22] Y. J. Kim and E. P. Merkes, "On an Integral of Powers of a Spirallike Function," Kyungpook Mathematical Journal, Vol. 12, No. 2, 1972, pp. 249-252.

[23] N. N. Pascu and V. Pescar, "On the Integral Operators of Kim-Merkes and Pfaltzgraff," Mathematica, Universitatis Babes-Bolyai Cluj-Napoca, Vol. 32, No. 2, 1990, pp. 185192. 